\title{
Diffuse gingival enlargement with stromal calcifications occurring in a background of amelogenesis imperfecta - An oral medicine case book
}

\author{
SADJ September 2020, Vol. 75 No. 8 p450 - p453
}

E Heyl'1, L Robinson², L Kotze ${ }^{3}$, WFP van Heerden ${ }^{4}$

\section{CASE REPORT}

A 12-year-old female patient presented with diffusely enlarged fibrous gingivae, enamel hypoplasia, an anterior open bite and impacted permanent maxillary canines (Figures 1-4). The patient's mother reported that the child had an unremarkable medical history and was currently not taking any medications. Radiographic examination showed features of amelogenesis imperfecta affecting all erupted teeth and the impacted permanent maxillary canines (Figure 4).

The clinical differential diagnosis included hereditary gingival fibromatosis or diffuse peripheral odontogenic fibromas involving both the maxilla and mandible. Gingivectomies from the anterior maxillary and mandibular regions were performed and submitted for histological assessment.

\section{DIAGNOSIS AND MANAGEMENT}

The gingivectomy specimens were submitted in two separate containers. The maxillary gingivectomy consisted of four soft tissue fragments, the largest measuring

\section{Author affiliations:}

1. Eric Heyl: Final Year Dentistry Student, School of Dentistry, Faculty of Health Sciences, University of Pretoria, Pretoria, South Africa.

ORCID Number: 0000-0003-1527-0575

2. Liam Robinson: $B C h D, P D D$ (Maxillofacial Radiology), PDD (Forensic Odontology), Department of Oral Pathology and Oral Biology, School of Dentistry, Faculty of Health Sciences, University of Pretoria, Pretoria, South Africa.

ORCID Number: 0000-0002-0549-7824

3. Leande Kotze: $B C h D$, Department of Periodontics and Oral Medicine, School of Dentistry, Faculty of Health Sciences, University of Pretoria. ORCID Number: 0000-0003-1518-4941

4. Willie FP van Heerden: BChD, MChD, FC Path (SA) Oral Path, PhD, $D S c$, Department of Oral Pathology and Oral Biology, School of Dentistry, Faculty of Health Sciences, University of Pretoria. ORCID Number: 0000-0003-2494-667X

Corresponding author: Willie FP van Heerden

Department of Oral Pathology and Oral Biology, University of Pretoria, Pretoria, South Africa.

Email: willie.vanheerden@up.ac.za

Author contributions:

1. Eric Heyl: Principle author $-50 \%$

2. Liam Robinson: Diagnosis, histological images, advisor $-20 \%$

3. Leande Kotze: Clinical case, treatment and follow-up - 15\%

4. Willie FP van Heerden: Diagnosis, advisor - $15 \%$

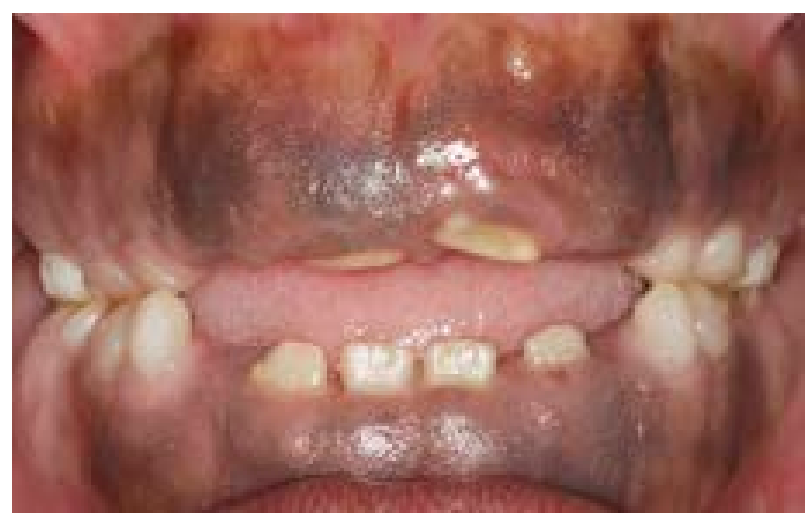

Figure 1. Initial clinical presentation. Diffusely enlarged fibrous gingivae obscuring the clinical crowns. Both maxillary central incisors show evidence of enamel hypoplasia.

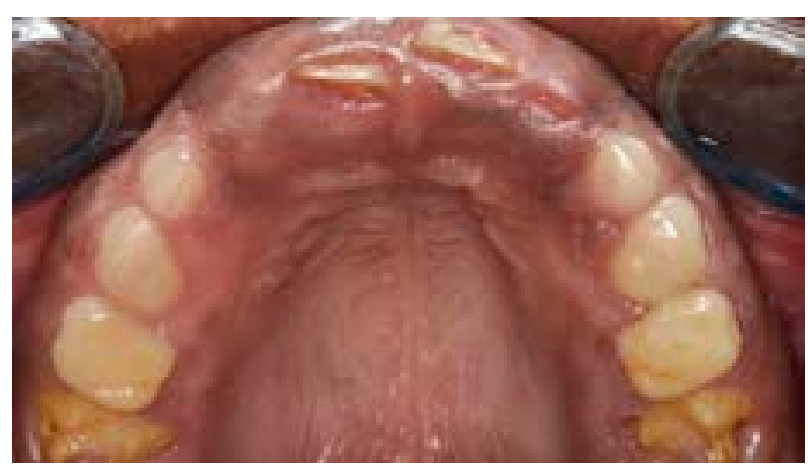

Figure 2. Maxillary arch. Diffuse gingival enlargement, particularly involving the anterior maxilla. Both permanent first maxillary molars show evidence of enamel hypoplasia.

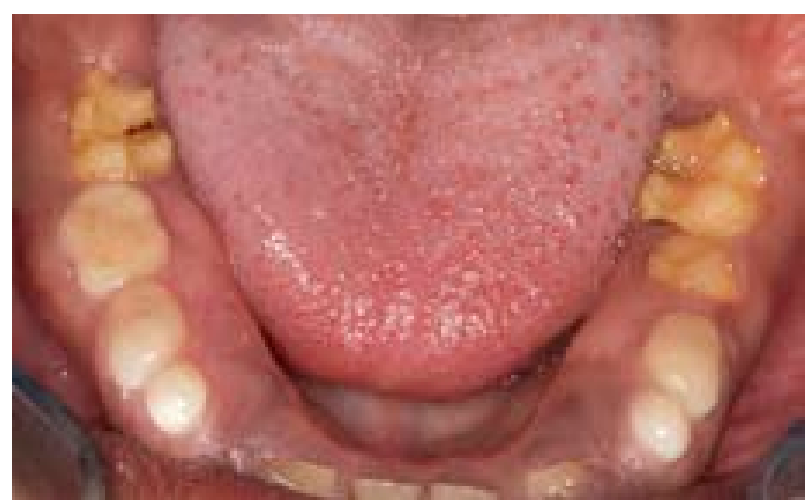

Figure 3. Mandibular arch. Diffuse gingival enlargement with evidence of enamel hypoplasia involving both permanent first mandibular molars. 
$7 \times 5 \times 3 \mathrm{~mm}$ and the smallest measuring $4 \times 4 \times 2 \mathrm{~mm}$. The mandibular gingivectomy consisted of two soft tissue fragments, the larger measuring $20 \times 5 \times 3 \mathrm{~mm}$ and the smaller measuring $10 \times 3 \times 2 \mathrm{~mm}$.

Histological examination of both specimens showed similar features, confirming tissue fragments surfaced by mildly hyperplastic stratified squamous epithelium (Figure 5). The superficial lamina propria consisted of dense fibrous connective tissue with a patchy mixed chronic inflammatory cell infiltrate of moderate intensity.
Additionally, inactive odontogenic epithelial islands with associated irregular and laminated dystrophic calcifications were noted (Figure 5 insert). There was representation of the junctional epithelium with extensive inflammatory cell exocytosis. A Periodic acid-Schiff (PAS) histochemical stain failed to highlight any fungal elements.

Considering the clinical, radiographic and histological features, a diagnosis of diffuse gingival enlargement with stromal calcifications occurring in a background of amelogenesis imperfecta was made. The patient was sche-

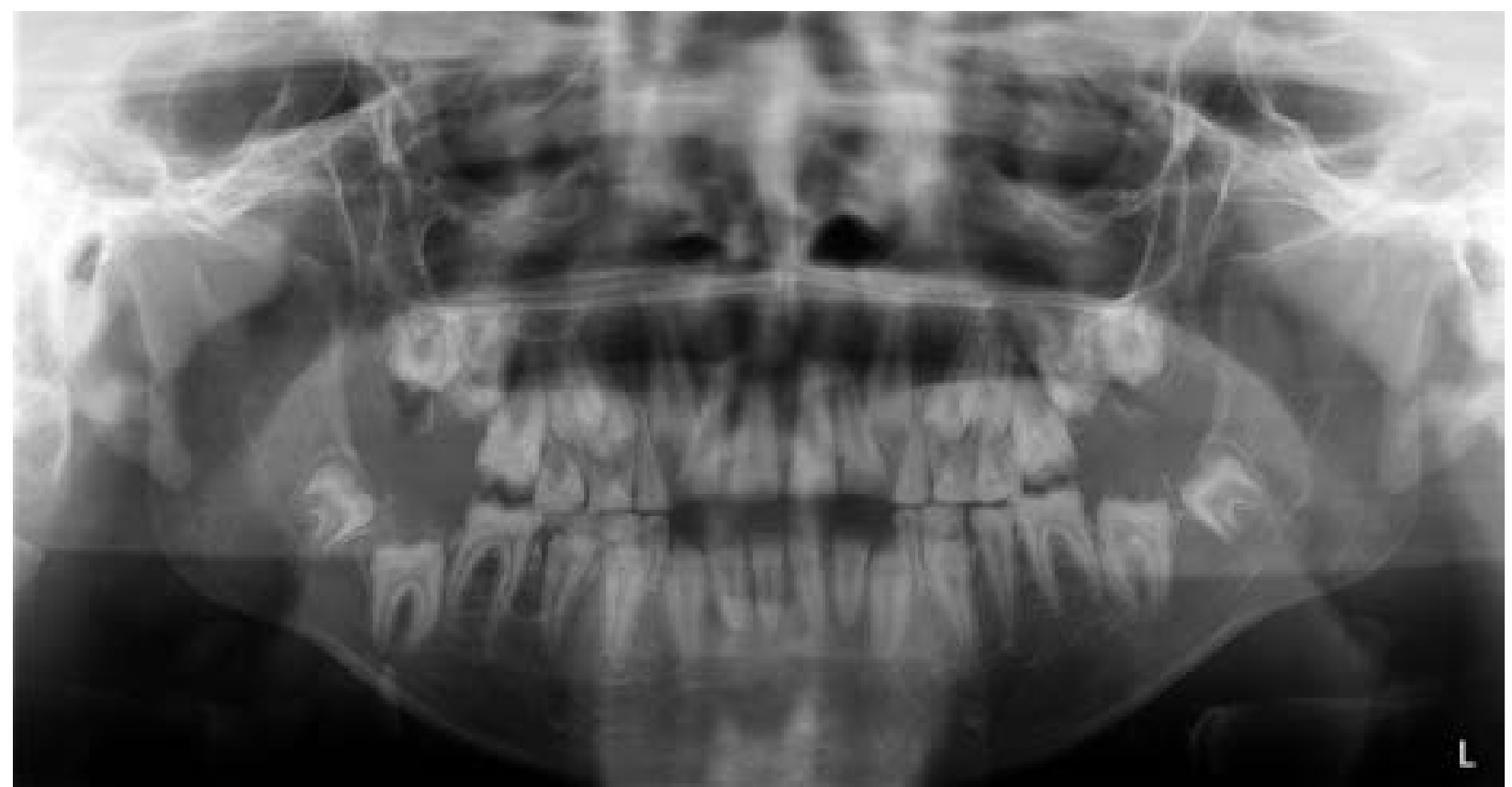

Figure 4. Panoramic radiograph showing diffuse enamel hypoplasia as well as bilateral impacted permanent maxillary canines. An incidental finding of unilocular radiolucency situated between teeth 45 and 46 was noted.

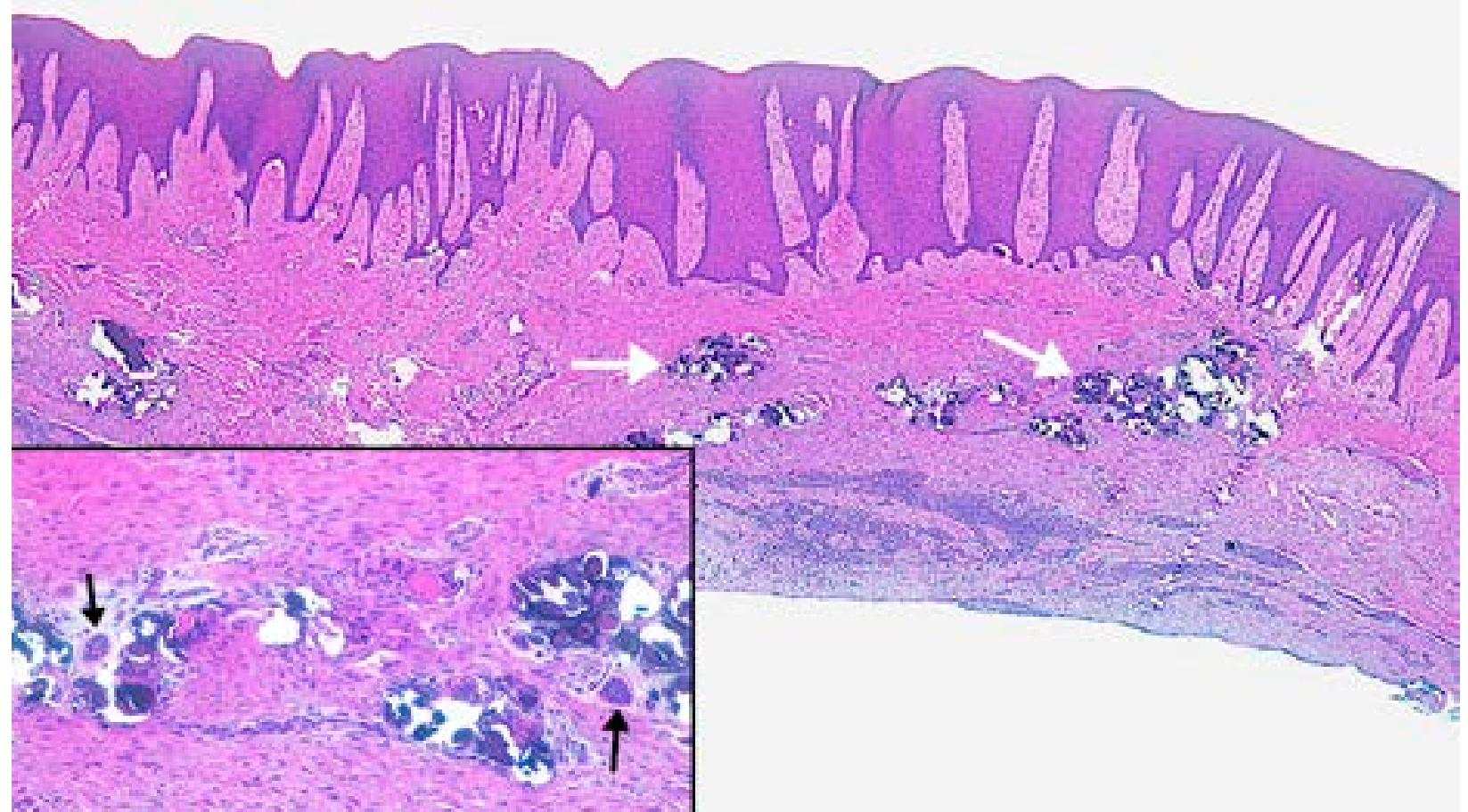

Figure 5. A low-power hematoxylin and eosin (H\&E)-stained section showing showing a tissue fragment surfaced by hyperplastic stratified squamous epithelium with inactive odontogenic epithelial islands with associated irregular and laminated dystrophic calcifications (white arrows) within the lamina propria (original magnification $x$ 40). Insert: High-power view of the odontogenic epithelial islands (black arrows) and calcifications (original magnification $\times 200)$. 
duled for a follow-up appointment one month post periodontal surgery to evaluate healing and to assess for further treatment. The proposed treatment of this patient included dental hard tissue rehabilitation using stainless steel crowns on the posterior teeth and composite strip crowns on the anterior teeth.

\section{DISCUSSION}

Gingival diseases frequently present as gingival enlargement, but have a wide range of presentations that can pose a diagnostic challenge to the clinician. Gingival enlargement can be characterised based on location, size, distribution, and aetiopathogenesis. ${ }^{1}$ After detailed examination and exclusion of unlikely differential diagnosis, a final diagnosis can be made in most instances. However, some cases require additional clinical work-up including a biopsy for a definitive histological diagnosis.

There are many causes of diffuse gingival enlargement including poor oral hygiene, systemic medications, genetic conditions, neoplasia, serious systemic illnesses and other specific physiological states. ${ }^{2}$ In some instances the underlying cause of the diffuse enlargement cannot be determined and is therefore considered idiopathic. ${ }^{2}$ Although diffuse gingival enlargement is considered a relatively common finding, it can be a pertinent clinical clue of an underlying serious systemic condition. ${ }^{1-2}$

Amelogenesis imperfecta (Al) is a hereditary condition of abnormal enamel calcification and formation. The disease encompasses a complicated group of disorders that demonstrate developmental alterations in the structure of enamel, in the absence of a systemic disease or syndrome. ${ }^{3}$ Although this definition may exclude the association with a syndrome, a number of other dental abnormalities may be seen in patients with Al. These include pulpal calcifications, anterior open bite malocclusion, taurodontism, gingival enlargement and delayed eruption. ${ }^{3} \mathrm{~A}$ wide range of hereditary subtypes exist with different patterns of inheritance and an array of clinical manifestations.

Although an ideal classification system for Al has not yet been established, the Witkop classification appears to be the most accepted. ${ }^{4}$ Simplified, Al may be sub classified into four main forms, depending on the type and extent of enamel defects. The presentation may be hypoplastic, hypomaturation, hypocalcified or hypomaturation/hypoplastic with associated taurodontism. . $^{3-4}$

Several gene mutations have been associated with the development of Al. Diverse and distinctive phenotypic patterns are often created due to variations in individual gene mutations. To date, commonly mutated genes include: AMELX associated with the enamel protein amelogenin, ENAM associated with the enamel matrix protein enamelin, MMP-20 which codes for proteinase enamelysin, KLK4 coding for the protease kallikrein-4, C4orf26 which encodes extracellular matrix protein in the enamel organ, and $D L X 3$ which codes for a number of essential enamel proteins. In addition, certain gene mutations have been associated with specific forms of Al. FAM83H gene mutations have been associated with autosomal-dominant hypocalcified Al, whereas
WDR72 gene mutations have been associated with autosomal-recessive hypomaturation $\mathrm{Al}^{3-4}$

Literature provides reasonable evidence linking amelogenesis imperfecta and diffuse gingival enlargement. A 1992 case report by Peters et al. suggested that there may be an association between Al and hereditary gingival fibromatosis (HGF) on the basis of sibling hereditary patterns, clinical, radiological and histological findings. ${ }^{5}$ Another common trend in the literature postulates that the gingival enlargement associated with $\mathrm{Al}$ is attributed to the rough hypoplastic nature of the remaining tooth surface enamel which promotes microscopic bacterial plaque accumulation and retention, leading to a chronic inflammatory host response. ${ }^{6}$ This hypothesis is justified by the inflammatory cell infiltrate, dense fibrous connective tissue and dystrophic calcifications noted histologically, however these findings can also be seen in HGF. 6

A study by Cherkaoui Jaouad et al. confirmed that mutations in the FAM20A gene are responsible for the development of autosomal-recessive $\mathrm{Al}$ with concurrent gingival fibromatosis. ${ }^{7}$ This may validate the presence of gingival enlargement in the background of Al from a familial genetic perspective. Further studies are needed to determine the exact mechanism of gingival overgrowth associated with $\mathrm{Al}$, and whether the source of the overgrowth is as a result of fibroblast activity or human growth factors. ${ }^{8-9}$

The presence of irregular, laminated dystrophic calcifications in the gingiva adjacent to inactive odontogenic epithelial islands in patients with known Al is an uncommon and rarely described phenomenon. In 1995, Günhan et al. described the occurrence of multiple dystrophic calcifications, deposition of amyloid and islands of odontogenic epithelium in the gingiva of three related siblings diagnosed with familial gingival fibromatosis. ${ }^{10}$ The calcifications resembled those found in the hyperplastic dental follicles of regional odontodysplasia. ${ }^{10}$

A case report by Macedo et al. presented an association between hypoplastic type $\mathrm{Al}$ and diffuse gingival enlargement. ${ }^{6}$ In their report, histological examination of the gingival specimen showed dense fibrous connective tissue with a mononuclear inflammatory cell infiltrate and dystrophic calcifications with odontogenic epithelial islands. Rao and Carnelio reported an additional case of gingival enlargement with calcifications in the background of $\mathrm{Al}$ in a 12-year-old female presenting with hypoplastic enamel and gingival hyperplasia, without a family history of this condition. ${ }^{11}$ A diagnosis of autosomal-recessive hypoplastic Al of the rough variant was established based on clinical and radiographic examinations. On histological examination, calcifications with either a concentrically lamellated or globular pattern were present in the hyperplastic gingival specimen. These calcifications, referred to as 'enameloid conglomerates', have been described in several types of $\mathrm{Al}$ and also in regional odontodysplasia. ${ }^{11}$ The authors further attempted to determine the character of these calcifications via histochemical stains, concluding that they were derived from odontogenic epithelial rests that had undergone dystrophic calcification. ${ }^{11}$ 
Regional odontodysplasia (ROD) often shows dystrophic calcifications with laminated or globular morphologies and islands of odontogenic epithelium in the follicular tissue surrounding the crown. ${ }^{12}$ ROD is a rare condition defined as nonhereditary amelogenesis imperfecta. It typically affects a focal area of the dentition with an affinity for the anterior teeth, and exhibits no gender or racial predilection. ${ }^{12}$ Radiographically, the enamel and dentine appear thin with an enlarged pulp chamber. This radiographic appearance is often termed 'ghost teeth' owing to the lack of enamel-dentine contrast. Delayed eruption, non-inflammatory gingival enlargement and short roots with open apices are common clinical manifestations of ROD. Of the teeth that do erupt, many exhibit discoloured yellow/brown crowns with irregular enamel, rendering them particularly susceptible to dental caries. ${ }^{12}$ Diagnosis is usually based on clinical and radiographic examination, with occasional histologic confirmation. Histologically, ROD displays varying degrees of dystrophic pulpal calcifications. .,12 $^{3}$

Amelogenesis imperfecta that occurs with gingival enlargement resembling HGF is generally managed via periodontal flap surgery. This has shown relative success in restoring the normal physiology of the gingiva. ${ }^{13-14}$ This is considered an adjunct to the restorative management, which is subdivided into different phases, depending on the eruption status of the patient. ${ }^{13-15}$

The temporary phase is performed during primary and mixed dentitions. In the primary dentition, stainless steel crowns are placed on molars with the purpose of preventing caries while allowing for adequate space maintenance and occlusal vertical dimension (OVD). Direct composite resins can be used on anterior teeth for aesthetic purposes. In the mixed dentition, stainless steel crowns, composite resin restorations or onlays on posterior teeth, and orthodontics are the conservative approach of choice to maintain OVD. ${ }^{15}$ Direct or indirect composite resin veneers may be used to improve dental aesthetics. In the mixed dentition, the main goal is to preserve of tooth structure, maintain tooth vitality, reduce tooth sensitivity, maintain the OVD and improve aesthetics. $^{15}$

The transitional phase begins when all permanent teeth have erupted and continues till adulthood, whereby the permanent phase begins. In permanent teeth, full mouth rehabilitation with a multidisciplinary approach including prosthodontics, endodontics, periodontics and orthodontics may be necessary. ${ }^{15}$

Treatment may also include orthognathic surgery and orthodontic treament in cases of severe malocclusion. Endodontic treatment may be necessary in cases of pulp exposure due to severe attrition or tooth reduction. Consultation with the appropriate specialists may help in developing a comprehensive treatment plan. ${ }^{15}$

\section{CONCLUSION}

The aetiology of diffuse gingival enlargement is diverse, including poor oral hygiene, systemic medications, genetic conditions, neoplasia, serious systemic illnesses and other specific physiological states. There is reasonable evidence linking amelogenesis imperfecta and diffuse gingival enlargement. ${ }^{6,11}$ In these cases, periodontal flap surgery can help restore the normal physiology of the gingiva. ${ }^{13-14}$ Although rare, several case reports describe dystrophic calcifications in gingivectomy specimens of patients with Al additionally presenting with diffuse gingival enlargment.

The restorative management of $\mathrm{Al}$ is subdivided into different phases, depending on the eruption status of the patient. The main objective of this treatment is to preserve tooth structure to maintain vitality and improve overall aethestics. ${ }^{15} \mathrm{~A}$ comprehesive treatment plan, developed via a multidisciplinary approach, is necessary to provide optimal treatment for these patients.

\section{References}

1. Agrawal AA. Gingival enlargements: Differential diagnosis and review of literature. World J Clin Cases. 2015; 3(9): 779-88.

2. Khera P, Zirwas MJ, English JC. Diffuse gingival enlargement. J Am Acad Dermatol. 2005; 52 (3 Pt 1): 491-9.

3. Neville BW, Damm DD, Allen CM, Chi AC. Oral and Maxillofacial Pathology. Fourth edition. St. Louis, MO: Elsevier; 2016.

4. Witkop CJ, Jr. Amelogenesis imperfecta, dentinogenesis imperfecta and dentin dysplasia revisited: Problems in classification. J Oral Pathol. 1988; 17(9-10): 547-53.

5. Peters E, Cohen M, Altini M. Rough hypoplastic amelogenesis imperfecta with follicular hyperplasia. Oral Surg Oral Med Oral Pathol. 1992; 74(1): 87-92.

6. Macedo GO, Tunes RS, Motta AC, Passador-Santos F, Grisi MM, Souza SL, et al. Amelogenesis imperfecta and unusual gingival hyperplasia. J Periodontol. 2005; 76(9): 1563-6.

7. Cherkaoui Jaouad I, El Alloussi M, Chafai El Alaoui S, Laarabi FZ, Lyahyai J, Sefiani A. Further evidence for causal FAM20A mutations and first case of amelogenesis imperfecta and gingival hyperplasia syndrome in Morocco: a case report. BMC Oral Health. 2015; 15: 14.

8. Gokce K, Canpolat C, Ozel E. Restoring function and esthetics in a patient with amelogenesis imperfecta: A case report. J Contemp Dent Pract. 2007; 8(4): 95-101.

9. Akin H, Tasveren S, Yeler DY. Interdisciplinary approach to treating a patient with amelogenesis imperfecta: A clinical report. J Esthet Restor Dent. 2007; 19(3): 131-5; discussion 6.

10. Gunhan O, Gardner DG, Bostanci H, Gunhan M. Familial gingival fibromatosis with unusual histologic findings. J Periodontol. 1995; 66(11): 1008-11.

11. Rao N, Carnelio S. Amelogenesis imperfecta with gingival calcification: A rare presentation. Brazilian Journal of Oral Sciences. 2015; 4(15): 932-5.

12. Kappadi D, Ramasetty PA, Rai KK, Rahim AM. Regional odontodysplasia: An unusual case report. J Oral Maxillofac Pathol. 2009; 13(2): 62-6.

13. Brennan MT, O'Connell BC, Rams TE, O'Connell AC. Management of gingival overgrowth associated with generalized enamel defects in a child. J Clin Pediatr Dent. 1999; 23(2): 97-101.

14. Yaprak E, Subasi MG, Avunduk M, Aykent F. Amelogenesis imperfecta and generalized gingival overgrowth resembling hereditary gingival fibromatosis in siblings: A case report. Case Rep Dent. 2012; 2012: 428423.

15. Chen CF, Hu JC, Bresciani E, Peters MC, Estrella MR. Treatment considerations for patient with amelogenesis imperfecta: A review. Braz Dent Sci. 2013; 16(4): 7-18. 